\title{
Periodic radio morphology of gamma-ray binaries
}

\author{
Javier Moldón ${ }^{* a b}$, Marc Ribó $^{b}$ and Josep M. Paredes ${ }^{b}$ \\ ${ }^{a}$ ASTRON Netherlands Institute for Radio Astronomy \\ Oude Hoogeveensedijk 4, 7991 PD Dwingeloo, The Netherlands \\ ${ }^{b}$ Departament d'Astronomia i Meteorologia, Institut de Ciències del Cosmos (ICC), Universitat \\ de Barcelona (IEEC-UB) \\ Martí i Franquès 1, 08028 Barcelona, Spain \\ E-mail: moldondastron, mribedam.ub.es, Gmparedesdub.edu
}

\begin{abstract}
Gamma-ray binaries allow us to study physical processes such as particle acceleration up to veryhigh energies and gamma-ray emission and absorption with changing geometrical configurations on a periodic basis. These sources produce outflows of radio-emitting particles whose structure can be imaged with Very Long Baseline Interferometry (VLBI). We have studied the changing morphology of the gamma-ray binaries LS I +61 303 and LS 5039, and we have discovered the extended emission of PSR B1259-63 and HESS J0632+057. Based on these results, we have established the basic properties and behaviour of the radio emission of gamma-ray binaries on AU scales, and we have contributed to find characteristics that are common to all of them. Here we present the most relevant properties of each source and the general properties of gamma-ray binaries, and we describe the implications on the nature of these binary systems.
\end{abstract}

11th European VLBI Network Symposium \& Users Meeting,

October 9-12, 2012

Bordeaux, France

${ }^{*}$ Speaker. 


\section{Introduction}

Some galactic binary systems have been detected in high-energy (HE; > $100 \mathrm{MeV}$ ) and/or very-high-energy (VHE; $>100 \mathrm{GeV}$ ) gamma rays (see [W] and references therein). Among these systems, gamma-ray binaries show a broadband non-thermal spectral energy distribution from radio to gamma-rays that is dominated by $\mathrm{MeV}-\mathrm{GeV}$ photons []. The spectral and brightness properties appear to be synchronised with the orbit of the binary system, which suggests that the physical conditions are also periodic and reproducible, and they do not show evidence of the presence of an accretion disc. The wide range of different orbital periods and eccentricities of the known gamma-ray binaries (see [3] ) provides a diversity of different ambient conditions in which the physical processes take place. The diversity of systems, together with the reproducibility of the conditions within each system, makes gamma-ray binaries excellent physical laboratories in which high-energy particle acceleration, diffusion, absorption, and radiation mechanisms can be explored. Nevertheless, the number of known gamma-ray binaries is still very limited.

Five binary systems have been classified as gamma-ray binaries: PSR B1259-63, LS 5039, LS I +61 303, HESS J0632+057, and 1FGL J1018.6-5856 [W]. The latter has not been clearly detected at $\mathrm{TeV}$ energies yet. In all of them the optical companion is a young massive star. The VHE gamma-ray emission can be interpreted as the result of inverse Compton upscattering of stellar UV photons by relativistic electrons, although hadronic models do exist as well. The acceleration of electrons can be explained by two excluding scenarios: acceleration in the jet of a microquasar powered by accretion [四], or shocks between the relativistic wind of a young non-accreting pulsar and the wind of the stellar companion [■, [1, 目]. Gamma-ray binaries allow us to study physical processes such as particle acceleration up to very-high energies and gamma-ray emission and absorption with changing geometrical configurations on a periodic basis.

Gamma-ray binaries are known to display non-thermal synchrotron radio emission of the order of 0.1-100 milliJansky (mJy). Relativistic electrons that travel away from the system can produce radio emission up to distances of several AU. Therefore, the radio outflows can easily reach projected angular separations of the order of milliarcseconds (mas), which are directly observable by means of Very Long Baseline Interferometry (VLBI) at radio wavelengths.

\section{Extended radio emission in gamma-ray binaries}

Four gamma-ray binaries have been observed with VLBI. We describe the main properties of the systems and the results from our recent and new VLBI observations.

PSR B1259-63 The binary system PSR B1259-63/LS 2883 is formed by a young 48 ms radio pulsar in an eccentric orbit of 3.4 years around a massive main-sequence star [ $[0]$. The spectral type of the massive star, $09.5 \mathrm{Ve}$, and some of the binary parameters have been recently updated by [8]], who obtained a distance to the system of $2.3 \pm 0.4 \mathrm{kpc}$. PSR B1259-63 is the only gamma-ray binary in which radio pulsations have been detected up to now.

We observed PSR B1259-63 with the Australian Long Baseline Array (LBA) at $2.3 \mathrm{GHz}$ in 2007 during three epochs corresponding to $1.3,21.2$, and 315.5 days after the periastron passage [Q]. In the first two epochs the radio source showed extended emission up to projected distances of 


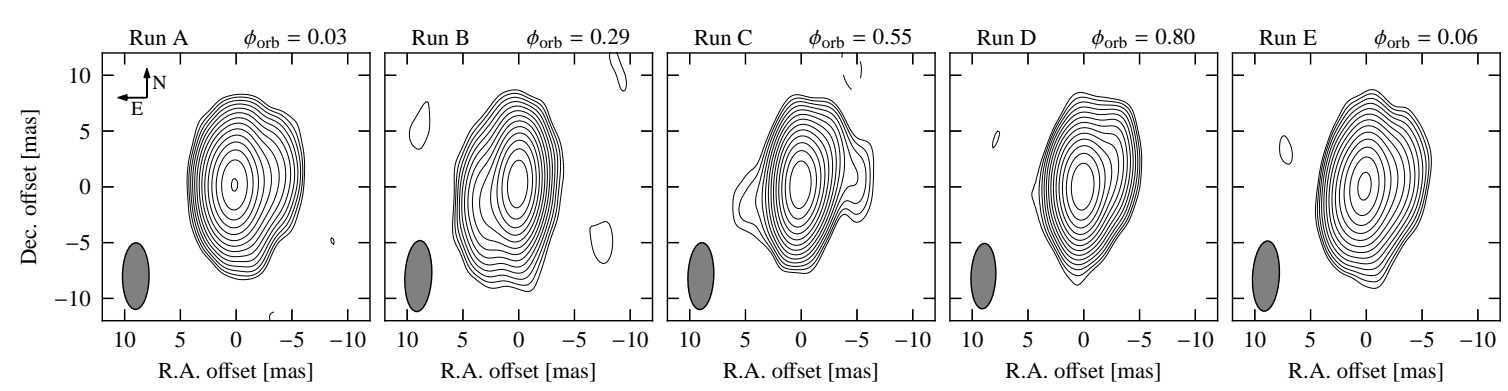

Figure 1: VLBA images of LS 5039 at $5 \mathrm{GHz}$ from project BR127, obtained during five consecutive days in July 2007 [प]4]. Each column, labelled with the run name and orbital phase, corresponds to one epoch. The self-calibrated images were produced using a natural weighting scheme. The restoring beams are plotted in the bottom-left corner of each panel. Dashed contours are plotted at -3 times the rms noise of each image and solid contours start at 3 times the rms and scale with $2^{1 / 2}$.

$\sim 50$ mas, or $\sim 120 \mathrm{AU}$. The third epoch, obtained closer to the apastron passage, showed a faint point-like source, which was interpreted as emission from the pulsar that indicates the position of the binary system. The position of the binary system within the nebula for the first two epochs was estimated using the size of the orbit and the proper motion of the system. The results showed that the peak of the radio emission is displaced from the binary system. Preliminary VLBI images of the 2010 periastron passage of PSR B1259-63 suggest that its VLBI structure varies periodically (Moldón et al., in preparation).

LS 5039 This system, located at $2.9 \pm 0.8 \mathrm{kpc}$, is composed by an ON6.5 V((f)) massive star and a compact object of unknown mass in a $3.9 \mathrm{~d}$ orbit [ए]] . The radio emission appears extended when observed with VLBI on mas scales. At $5 \mathrm{GHz}$, the source shows a main core and extended bipolar emission that has been detected at projected angular distances between 1 and 180 mas (3-500 AU) from the core $[\mathbb{[}-[\mathrm{\square} 3]$.

We observed LS 5039 with the VLBA at $5 \mathrm{GHz}$ during five consecutive days, covering a whole orbital cycle [14]]. The resulting self-calibrated images are shown in Fig. $\mathrm{W}$. The radio morphology on mas scales is variable, with changes in less than 24 hours. Images at similar orbital phases show a similar morphology. We found that this behaviour is stable on time scales of years. Therefore, the gamma-ray binary LS 5039 shows periodic orbital morphological variability. We computed a model of the evolution of an outflow of relativistic electrons accelerated as a consequence of the interaction of the massive star and the wind of a young non-accreting pulsar. The model accounts for the main morphological features of LS 5039 [प14].

LS I +61 303 This gamma-ray binary contains a rapidly rotating B0e main sequence star with a stable equatorial shell, and a compact object orbiting it every $26.5 \mathrm{~d}$ in an eccentric orbit with $e=$ 0.72 [ए5]]. It displays quasi-periodic radio outbursts at radio, X-rays and gamma-rays modulated by a long-term periodicity of $\sim 4.6 \mathrm{yr}$ [ए6]. [ए]] conducted several VLBI observations showing an orbital variability that these authors interpreted as the signature of a cometary tail produced in the colliding winds scenario. Another interpretation of the data suggests that the changes are compatible with a precessing microblazar [1[8]. 


\begin{tabular}{lcccc}
\hline Source & $P_{\text {orb }}[\mathrm{d}]$ & Observed & VLBI structure & Variability \\
\hline LS 5039 & 3.9 & Yes & Yes & Periodic changes \\
1FGL J1018.6-5856 & 16.6 & No & $?$ & - \\
LS I +61 303 & 26.5 & Yes & Yes & Periodic changes \\
HESS J0632+057 & 321 & Yes & Yes & Variable \\
PSR B1259-63 & 1236.8 & Yes & Yes & Periodic changes \\
\hline
\end{tabular}

Table 1: Summary of the VLBI observations of the known gamma-ray binaries.

We observed LS I +61 303 with the VLBA in 2007 at 8.4 and $2.3 \mathrm{GHz}$ during five epochs separated by two days and covering an $\mathrm{X}$-ray/TeV outburst. We found extended and variable emission on scales of 5-10 AU. The observed morphology is compatible with the one found in observations conducted one year before at similar orbital phases, which suggests that the periodic orbital modulation is stable on time scales of years. We obtained accurate astrometry at two radio frequencies and found the relative offsets between them. The behaviour found is similar to the one expected from an outflow whose axis passes close to the line of sight of the observer (Moldón et al., in preparation).

HESS J0632+057 The proposed optical counterpart of HESS J0632+057 is the massive B0pe star MWC $148[\mathbb{L}]$. A periodicity of $321 \pm 5 \mathrm{~d}$ was revealed by long-term X-ray observations conducted with Swift/XRT [20]. MWC 148 was confirmed to be a binary system by means of radial velocity observations [B]]. The radio counterpart is variable, although no detailed radio light curve is available.

We observed HESS J0632+057 with the EVN at $1.6 \mathrm{GHz}$ in ToO mode in two epochs: February 15 and March 17, 2011, following the report of an X-ray outburst. The first observation (eEVN) was conducted 9 days after the peak of the X-ray outburst, whereas the second observation (full EVN) was conducted 30 days later. The source is point-like during run A and displays extended emission up to projected distances of $75 \mathrm{AU}$ in run B [R]]. The peak of the emission is displaced $21 \mathrm{AU}$ between runs. The radio counterpart of HESS J0632+057 is unambiguously identified with MWC 148.

1FGL J1018.6-5856 For completeness, a short comment on this new gamma-ray binary candidate. 1FGL J1018.6-5856 was discovered by searching for periodicities of Fermi sources, and shows intensity and spectral modulation at $\mathrm{GeV}$ energies with a 16.6-day period [ए2]. The proposed optical counterpart is an $\mathrm{O} 6 \mathrm{~V}((\mathrm{f}))$ star. The source has a variable flux density of $2-5 \mathrm{mJy}$ as measured with Australia Telescope Compact Array (ATCA), although no extended emission has been reported so far.

As a summary, in Table $\square$ we show the list of known gamma-ray binaries with the orbital period of the system, and the properties of the VLBI structure of each source. Periodic morphological changes have been seen in LS 5039, LS I +61 303, and PSR B1259-63, whereas for the recently discovered sources HESS J0632+057 and 1FGL J1018.6-5856, more data is needed in order to obtain evidence of periodicity. 


\section{Conclusions}

The four gamma-ray binaries detected at $\mathrm{TeV}$ energies show extended structures when observed with VLBI. The common properties of the radio structures from PSR B1259-63, LS 5039, and LS I +61 303 can be summarized in three points, which are model independent: (1) They display synchrotron radio emission that forms structures on AU scales. (2) These radio structures show periodic orbital morphological variability, although erratic changes might occur. (3) The position of the peak of the emission suffers displacements significantly larger than the size of the orbit. For HESS J0632+057, the second property has not been verified yet due to the lack of observations during different orbital cycles. In summary, the VLBI data obtained in recent years for the known gamma-ray binaries show that most of them display periodic morphological changes, coupled with the orbital period, and therefore this behaviour is expected for all gamma-ray binaries.

\section{Acknowledgments}

J.M., M.R., and J.M.P. acknowledge support by MINECO under grants AYA2010-21782-C0301 and FPA2010-22056-C06-02. M.R. acknowledges financial support from MINECO and European Social Funds through a Ramón y Cajal fellowship. J.M.P. acknowledges financial support from ICREA Academia.

\section{References}

[1] J. M. Paredes, "Gamma-ray binaries: microquasars and binary systems with pulsar," in SciNeGHE 2010 (2011).

[2] G. Dubus, Astronomy and Astrophysics 456, 801-817 (2006).

[3] J. Casares, M. Ribó, I. Ribas, J. M. Paredes, F. Vilardell, and I. Negueruela, Monthly Notices of the RAS 421, 1103 (2012a).

[4] V. Bosch-Ramon, G. E. Romero, and J. M. Paredes, Astronomy and Astrophysics 447, 263-276 (2006).

[5] L. Maraschi, and A. Treves, Monthly Notices of the RAS 194, 1P-5P (1981).

[6] D. Khangulyan, S. Hnatic, F. Aharonian, and S. Bogovalov, Monthly Notices of the RAS 380, 320-330 (2007).

[7] S. Johnston, R. N. Manchester, A. G. Lyne, L. Nicastro, and J. Spyromilio, Monthly Notices of the RAS 268, 430 (1994).

[8] I. Negueruela, M. Ribó, A. Herrero, J. Lorenzo, D. Khangulyan, and F. A. Aharonian, Astrophysical Journal, Letters 732, L11 (2011).

[9] J. Moldón, S. Johnston, M. Ribó, J. M. Paredes, and A. T. Deller, Astrophysical Journal, Letters 732, L10 (2011a).

[10] J. Casares, J. C. Morales, A. Herrero, I. Ribas, M. Ribó, J. M. Paredes, J. M. Corral-Santana, and T. Muñoz-Darias, Astronomy and Astrophysics, submitted (2012).

[11] J. M. Paredes, J. Martí, M. Ribó, and M. Massi, Science 288, 2340-2342 (2000). 
[12] J. M. Paredes, M. Ribó, E. Ros, J. Martí, and M. Massi, Astronomy and Astrophysics 393, L99-L102 (2002).

[13] M. Ribó, J. M. Paredes, J. Moldón, J. Martí, and M. Massi, Astronomy and Astrophysics 481, 17-20 (2008).

[14] J. Moldón, M. Ribó, and J. M. Paredes, Astronomy and Astrophysics 548, A103 (2012).

[15] J. Casares, I. Ribas, J. M. Paredes, J. Martí, and C. Allende Prieto, Monthly Notices of the RAS 360, 1105-1109 (2005).

[16] P. C. Gregory, Astrophysical Journal 575, 427-434 (2002).

[17] V. Dhawan, A. Mioduszewski, and M. Rupen, "LS I +61 303 is a Be-Pulsar binary, not a Microquasar," in VI Microquasar Workshop: Microquasars and Beyond, 2006.

[18] M. Massi, E. Ros, and L. Zimmermann, Astronomy and Astrophysics 540, A142 (2012).

[19] J. A. Hinton, J. L. Skilton, S. Funk, J. Brucker, F. A. Aharonian, G. Dubus, A. Fiasson, Y. Gallant, W. Hofmann, A. Marcowith, and O. Reimer, Astrophysical Journal, Letters 690, L101-L104 (2009).

[20] S. D. Bongiorno, A. D. Falcone, M. Stroh, J. Holder, J. L. Skilton, J. A. Hinton, N. Gehrels, and J. Grube, Astrophysical Journal, Letters 737, L11 (2011).

[21] J. Moldón, M. Ribó, and J. M. Paredes, Astronomy and Astrophysics 533, L7 (2011b).

[22] Fermi LAT Collaboration, Ackermann, M., Ajello, M., et al., Science 335, 189 (2012). 